\title{
Desarrollo de la calidad de los indicadores de estructura física: un estudio nacional en los hospitales mentales públicos de Grecia
}

\author{
Michael G. Madianos, M.D. M.P.H. * \\ Costas Zacharakis, M.D. ** \\ Chryssa Tsitsa, MSc.**
}

* Professor of Psychiatry University of Athens School of Health Sciences, Faculty of Nursing

** Research Associate Monitoring and Evaluation of Mental Health Services Unit University Mental Health Research Institute

*** Statistician Monitoring and Evaluation of Mental Health Services Unit University Mental Health Research Institute

GREECE

RESUMEN - El estudio intentó desarrollar un instrumento para medir la calidad de la estructura física de los hospitales psiquiátricos públicos en Grecia.

El instrumento "The Conditions of Nursing: Dimensions of Inpatient Treatment" (CONDIT, Condiciones de los Cuidados de Enfermería: Dimensiones del Tratamiento Hospitalario) es original, adaptado culturalmente a las condiciones locales y contiene dos partes: el Departamento, y las plantas/unidades. El entrevistador valora los ítems de la escala, emitiendo juicios cualitativos sobre la condición física general del entorno y sus distintos componentes. Se evaluó la fiabilidad y validez del instrumento en 110 Departamentos (90\% del total), y en una muestra de 121 plantas de los nueve hospitales psiquiátricos públicos. El estudio de fiabilidad inter-examinadores obtuvo coeficientes de acuerdo kappa (k) estadísticamente significativos $(0,82)$. Además, tanto la consistencia interna (estimada con el coeficiente alfa de Cronbach) como la validez concurrente del CONDIT se consideraron muy satisfactorios. La aplicación del CONDIT documentó variabilidad significativa en los niveles de calidad de la estructura física entre los hospitales psiquiátricos públicos. Se consideró que el perfil global del entorno físico de las instituciones psiquiátricas públicas cumplía el criterio de "satisfactorio". 


\section{Introducción}

La garantía de calidad es un concepto amplio y puede definirse como un método para asegurar y mantener los aspectos de calidad asistencial de la salud en general y de la salud mental en particular, de acuerdo con los estándares, normas y criterios comúnmente aceptados (Donabedian 1966, Brugha y Lindsay 1996). Las áreas de la investigación en garantía de calidad son estructura, proceso y resultado, e incluyen varios factores administrativos, clínicos y económicos, así como relaciones interpersonales (Donabedian 1960, Fauman 1989).

Durante los últimos 20 años, en el campo de la psiquiatría y de la salud mental, un número creciente de publicaciones sobre garantía de calidad e investigación han producido un estado de conocimientos considerable en distintas áreas (Liptzin 1974, Wells y Brook 1988). La investigación sobre la garantía de calidad en salud mental coincide con un énfasis en el control de costes de los cuidados en psiquiatría, centrado principalmente en la medida de las dimensiones de estructura y proceso (Sherman 1987, Donabedian 1992). Pero rara vez se han evaluado los indicadores de resultados, debido a los costes de investigación y a los problemas y complicaciones en las definiciones de varios componentes, particularmente referidos a normas, criterios y estándares aceptables (Tugvel 1979, Schulberg 1976, Donabedian 1986). Como resultado del crecimiento de esta área de investigación, se han desarrollado varios sistemas de medición y algunos programas prácticos para la evaluación de los dominios de la garantía de calidad, y se han probado sus propiedades psicométricas (Davis y Fong 1996, Srebnik et al. 1997). Sorprendentemente, la investigación sobre estructura en la salud psiquiátrica pública en la era de la desinstitucionalización de los pacientes de larga estancia en muchos paí- ses no ha tenido como resultado el desarrollo de instrumentos y herramientas específicos comúnmente aceptados (Brugha y Lindsay 1996). Sin embargo, la Organización Mundial de la Salud ha publicado varias herramientas para la evaluación de la estructura en diferentes tipos de marcos, cubriendo las áreas de entorno físico, plantilla, limpieza, privacidad, etc. (Janka y Chandrashecar 1993).

Este trabajo se centra en los procedimientos llevados a cabo para el desarrollo de un instrumento que objetive la estructura física (material) de la estructura del hospital psiquiátrico público. Este instrumento se desarrolló para valorar la estructura física de los nueve hospitales psiquiátricos públicos en Grecia, en el contexto de un estudio sobre garantía de calidad nacional, llevado a cabo por la Unidad de Monitorización y Evaluación de los Servicios de Salud Mental del Instituto de Investigación en Salud Mental de la Universidad de Atenas.

La calidad de la estructura física de los hospitales psiquiátricos públicos griegos fue objeto de críticas tanto nacionales como internacionales a principios de los años 80 (Madianos 1983, Comisión de la Comunidad Europea 1984). En 1980, en Grecia, el sistema de provisión de cuidados en salud mental estaba centralizado, basado en los nueve Hospitales Psiquiátricos públicos atestados de pacientes, situados en el gran área de Atenas, Tesalónica, y en otras cinco áreas, con un total de 8.464 camas (Madianos 1983, Stefanis et al. 1986). Estas instituciones eran la mayoría de tipo manicomial, con una pobre organización de estructura, una plantilla inadecuada, y carentes de los necesarios programas de seguimiento y rehabilitación. Además de estos centros, la atención hospitalaria se llevaba a cabo en otros 40 hospitales psiquiátricos privados, con 5.393 camas (Madianos 1983).

Este estado de la salud mental en Grecia, y especialmente el caso bien conocido del 
Hospital Mental Leros, llamó la atención de la Comisión de la Comunidad Europea (CEC) a principios de los años 80 (Comisión de la Comunidad Europea 1984). En 1983, el gobierno griego revisó la organización del sistema de atención en salud mental y desarrolló un plan nacional, basado en el desarrollo de servicios de salud mental comunitarios descentralizados y de conjunto, y en la transformación de los hospitales psiquiátricos públicos existentes mediante la correspondiente desinstitucionalización de los pacientes ingresados de larga estancia (Madianos y Yfantopoulos 1990). En 1984, la implantación del programa de reforma psiquiátrica recibió apoyo económico y técnico excepcional de la CEC a través de la Regulación No 815/84, con una beca de 120 millones de ECU's para el periodo comprendido entre 1984 y 1995 (Madianos y Yfantopoulos 1990). Como resultado de este programa, se observó una reducción significativa del $42 \%$ de las camas de hospitales psiquiátricos entre 1984 y 1996 . Un prerrequisito básico para este apoyo económico de la CEC fue la evaluación y monitorización del progreso en la ejecución del programa de reforma psiquiátrica en Grecia.

Sin embargo, la evaluación y la investigación en servicios de salud mental fueron totalmente infra-desarrolladas, con pocas excepciones, principalmente en forma de informes anuales de los administradores de los hospitales psiquiátricos públicos, y una evaluación de las necesidades en el Hospital Mental de Leros (Madianos y Economou 1990, Tsiantis 1995). Por esta razón, el "Directorate General V" de la CEC fundó, en 1994, la Unidad de Monitorización y Evaluación de los Servicios de Salud Mental (MEMHSU) del Instituto de Investigación en Salud Mental de la Universidad de Atenas. En 1996, el MEMHSU tomó la iniciativa de evaluar las tres áreas de los indicadores de garantía de calidad en los hospitales psi- quiátricos públicos nacionales. Parte de los resultados de este estudio se describen en este artículo y, particularmente, el desarrollo y la estandarización de un instrumento para medir la estructura física de los hospitales psiquiátricos públicos.

\section{Método}

\section{Desarrollo del cuestionario}

Para la evaluación de las dimensiones cualitativas del entorno físico (material) de la estructura del hospital psiquiátrico público, y después de un estudio piloto de algunos cuestionarios existentes de la OMS, se detectaron varias inconsistencias culturales. De este modo, se decidió para la estructura un instrumento original llamado "The Conditions of Nursing: Dimensions of Inpatient Treatment" (CONDIT, Condiciones de los Cuidados de Enfermería: Dimensiones del Tratamiento Hospitalario). Se envió un borrador de este cuestionario a cada uno de los nueve directores médicos de los hospitales psiquiátricos públicos para obtener sus comentarios. Se recibieron respuestas de cinco hospitales con comentarios extensos y el cuestionario fue revisado de acuerdo con dichos resultados. Se revisó el cuestionario otras tres veces más hasta su versión final.

El cuestionario CONDIT contiene dos partes: la Sección de Departamento y la Sección de planta/unidad. El Departamento se define como una unidad autónoma desde el punto de la administración médico-clínica, con varias plantas/unidades de pacientes y otras actividades varias en el marco administrativo del hospital psiquiátrico. El CONDIT, en la sección de Departamento contiene 19 ítems divididos en cinco partes, que se refieren a: 1 . Condiciones generales del edificio (paredes, suelos, limpieza, luz, calefacción, aire acon- 
dicionado, teléfonos por paciente, seguridad contra incendios y seguridad contra gestos autolíticos; 2. Espacios de recreo (muebles, limpieza, ventilación, luz, receptores de TV, etc.); 3. Instalaciones de comedor (provisión de un comedor independiente, utensilios, limpieza); 4. Preparación de las comidas e instalaciones de almacenamiento; y 5. Espacios al aire libre (limpieza, jardín, bancos).

El CONDIT en la Sección de planta contiene 11 ítems denominados: espacio entre las camas, limpieza, cubrecamas, ventilación de la planta, luz natural, armarios, instalaciones de tuberías, condiciones de los aseos, duchas / pacientes y aseos / pacientes. Los ítems del cuestionario se puntúan mediante la observación in situ.

Para la Sección de Departamento del CONDIT, la puntuación 0-12 se refiere a niveles de condiciones "pobres". Las puntuaciones entre 13-28 y 28-38 reflejan niveles "satisfactorios" y "excelentes", respectivamente. Para la Sección de planta del CONDIT, la puntuación 0-6 corresponde a niveles de condiciones "pobres". Las puntuaciones 7-13 y 14-20 representan niveles "satisfactorios" y "excelentes" de condiciones físicas en las plantas, respectivamente.

\section{Desarrollo del estudio}

Las puntuaciones sobre las condiciones físicas de la estructura de los hospitales psiquiátricos públicos, tanto de Departamentos como de plantas las realizaron dos examinadores independientes. El estudio se llevó a cabo entre enero y febrero de 1998. Cuatro residentes de psiquiatría, tres psicólogos y siete trabajadores sociales visitaron los nueve hospitales psiquiátricos públicos, tras un entrenamiento de 25 horas y un estudio piloto de campo. Se completaron las evaluaciones en el $90 \%$ de los Departamentos psiquiátricos
(110) y en una muestra representativa de 121 plantas, mediante la aplicación del programa CONDIT y otros cuestionarios suplementarios.

\section{Estudio de fiabilidad}

Se llevó a cabo un estudio de fiabilidad inter-examinadores mediante la estimación del coeficiente kappa (k) de Cohen, de acuerdo entre los examinadores, sobre las puntuaciones CONDIT (Cohen 1960, Fleiss 1973). Se probó su consistencia interna mediante la estimación del coeficiente alfa de Cronbach.

\section{Estudio de validez}

Se midió la validez concurrente de la Sección de Departamento del programa CONDIT contrastándolo con el indicador más relevante, el del coste diario por paciente, descrito por el gestor económico de cada hospital psiquiátrico. Hay una relación directa entre el mantenimiento de la calidad del entorno físico de la estructura del hospital y el coste diario por paciente (Madianos 1996). El punto de corte entre coste alto y coste bajo diario por paciente lo establecieron los economistas del Ministerio de Salud. Los nueve hospitales fueron así agrupados en dos partes, en función de su coste diario por paciente (alto o bajo). Las diferencias entre las puntuaciones medias $\mathrm{CON}$ DIT en los distintos Departamentos se analizaron mediante la t de Student. El análisis estadístico se realizó aplicando el programa estadístico SPSS (Norusis 1983).

\section{Resultados}

En la Tabla I se muestran los resultados del estudio de fiabilidad del cuestionario 
CONDIT para el acuerdo entre dos examinadores independientes.

Se observó un acuerdo estadísticamente significativo $(p<0,001)$ entre los dos examinadores independientes referido a las puntuaciones sobre la calidad de las condiciones físi- cas de los Departamentos, con un coeficiente $\mathrm{k}=0,82$. Se encontró casi el mismo nivel de acuerdo entre los dos examinadores acerca de los niveles de calidad de las plantas (coeficiente $\mathrm{k}=0,81)$. Los coeficientes de correlación producto-momento $\left(\mathrm{r}_{\mathrm{s}}\right)$ de Pearson entre

Tabla I

Condiciones de los Cuidados de Enfermería: Dimensiones del Inventario de Tratamiento Hospitalario) (Sección de Departamento). Estudio de fiabilidad entre dos examinadores independientes

\begin{tabular}{lccccc} 
& Niveles de puntuación & \multicolumn{3}{c}{ Examinador A } \\
& & $0-12$ & $13-27$ & $28-38$ & $\mathrm{~N}$ \\
\hline Examinador B & $0-12$ & 18 & 1 & 2 & 19 \\
& $13-27$ & 2 & 72 & 11 & 76 \\
Total & $28-38$ & & 4 & 13 & 110 \\
\hline
\end{tabular}

Coeficiente kappa de Cohen: 0,825.

$\mathrm{P}<0,001$

Tabla II

Condiciones de los Cuidados de Enfermería: Dimensiones del Inventario de Tratamiento Hospitalario (Sección de Departamento). Estudio de fiabilidad, resultados de la consistencia interna

Coeficiente alfa

Condiciones generales del edificio

$\begin{array}{lr}\text { 1. Paredes, suelos, ventanas } & 0,86\end{array}$

2. Limpieza

0,86

3. Luz (artificial)

0,86

4. Calefacción

0,88

5. Aire acondicionado

0,88

6. Teléfonos para pacientes

0,88

7. Seguridad contra incendios

0,88

8. Previsiones de seguridad contra gestos autolíticos deliberados

Espacios de recreo

9. Muebles

0,87

10. Limpieza

0,86

11. Ventilación

0,86

12. Luz natural

0,87

13. Receptor de TV, juegos, etc.

0,87

Instalaciones de comedor

$\begin{array}{lr}\text { 14. Provisión de un comedor independiente } & 0,88\end{array}$

15. Utensilios

0,87

16. Limpieza

0,86

Preparación de las comidas e instalaciones de almacenamiento

17. Limpieza

0,87

Espacios al aire libre

18. Limpieza, orden

0,87

19. Bancos, jardines

0,88

Coeficiente alfa de Cronbach: 0,88. 
las puntuaciones CONDIT (en ambas secciones) entre los dos examinadores fueron incluso más altos $\left(\mathrm{r}_{\mathrm{s}}=0,87\right.$ y $\left.\mathrm{r}_{\mathrm{s}}=0,88, \mathrm{p}<0,0001\right)$.

También se documentó una alta consistencia interna del cuestionario mediante el coeficiente alfa de Cronbach (alfa $=0,88$ ) (Tabla II). Los valores alfa indican que la consistencia de los ítems del cuestionario es alta.

Como se muestra en la Tabla III, se encontró que la validez concurrente de la calidad de la estructura física de los Departamentos de los hospitales psiquiátricos con un coste diario alto por paciente era significativamente más alta que aquélla de los Departamentos con un coste diario bajo, en términos de valores medios de las puntuaciones CONDIT $(\mathrm{t}=2,16, \mathrm{p}<0,01)$.
Parece, pues, que la validez concurrente del cuestionario CONDIT es satisfactoria y que los ítems de la escala miden lo que pretenden medir.

En la Tabla IV, se presentan los rangos ordenados de las puntuaciones medias CONDIT (Sección de Departamento) para cada hospital psiquiátrico público. Según el sistema de puntuación del cuestionario, las puntuaciones bajas significan calidad pobre o inadecuada de las condiciones de la estructura del Departamento que se investiga. Los Departamentos psiquiátricos de los hospitales mentales de Attica y Corfú muestran las medias más bajas de las puntuaciones $\mathrm{CON}$ DIT, comparadas con las puntuaciones medias CONDIT de los otros hospitales. Los Departamentos del hospital psiquiátrico de

Tabla III

Validez concurrente del Inventario CONDIT (Sección de Departamento). Puntuaciones medias de dos grupos de hospitales psiquiátricos, según coste diario por paciente

\begin{tabular}{|c|c|c|c|c|c|c|}
\hline Coste diario & $\mathrm{N}^{\mathrm{o}}$ Hospitales & $\mathrm{N}^{\mathrm{o}}$ Departamentos & $\begin{array}{c}\text { Puntuación CONDIT } \\
\text { media (d.e.) }\end{array}$ & $\mathrm{t}$ & gl & $\mathrm{p}$ \\
\hline Alto & 4 & 53 & $22,01(5,47)$ & 2,18 & 108 & $<0,01$ \\
\hline Bajo & 5 & 57 & $19,70(5,75)$ & & & \\
\hline
\end{tabular}

gl: grados de libertad.

Tabla IV

Evaluación cualitativa de la estructura. Condiciones de los Cuidados de Enfermería: Dimensiones del Inventario de Tratamiento Hospitalario: Rangos ordenados de los valores globales medios en los Departamentos de los hospitales psiquiátricos públicos

\begin{tabular}{lrcrr} 
Hospital psiquiátrico público & $N^{\circ}$ Departamentos & $\begin{array}{c}\text { Puntuaciones } \\
\text { medias globales }\end{array}$ & d.e. & Rango \\
\hline 1. Thessaloniki Mental Hospital & 22 & 26,23 & 5,14 & $16-34$ \\
2. Chania Mental Hospital & 9 & 22,80 & 3,56 & $20-29$ \\
3. Attica Children's Mental Hospital & 8 & 22,12 & 5,48 & $13-28$ \\
4. Dromokaition Mental Hospital & 12 & 21,50 & 5,23 & $11-29$ \\
5. Petra Olympou Mental Hospital & 6 & 21,16 & 6,52 & $16-30$ \\
6. Leros Mental Hospital & 15 & 20,60 & 7,34 & $11-33$ \\
7. Tripolis Mental Hospital & 7 & 18,42 & 5,85 & $10-28$ \\
8. Corfu Mental Hospital & 5 & 17,00 & 5,29 & $12-23$ \\
9. Attica Mental Hospital & 26 & 16,75 & 6,27 & $6-26$ \\
Total & 110 & 20,63 & 5,63 & $20-29$ \\
\hline
\end{tabular}

Kruskal-Wallis 1 way anova $\mathrm{x}^{2}=55,93$ $\mathrm{p}<0,0001$. 
Tesalónica presentan las medias más altas de las puntuaciones CONDIT. Debe reseñarse que la puntuación media del programa CONDIT en los 15 Departamentos del hospital mental de Leros fue de 20,60 ( $\pm 7,34)$, casi la misma puntuación media que la del total de Departamentos del hospital.

Con respecto a la evaluación cualitativa de la estructura material de las Plantas, las plantas con alta puntuación CONDIT (niveles "satisfactorio" y "excelente") forman parte de los hospitales psiquiátricos de Leros, Tesalónica y Chania. Por el contrario, las plantas de los hospitales psiquiátricos de Corfú, Petra Olimpo y Trípoli obtuvieron las puntuaciones más bajas del cuestionario CONDIT, lo que significa pobres condiciones del entorno.

\section{Discusión}

Este estudio describe el desarrollo y las propiedades psicométricas de un instrumento para la evaluación de la calidad de las dimensiones estructurales físicas en los entornos asistenciales de los pacientes psiquiátricos hospitalizados. Particularmente, se diseñó el cuestionario "The Conditions of Nursing: Dimensions of Inpatient Treatment" (CONDIT, Condiciones de los Cuidados de Enfermería: Dimensiones del Tratamiento Hospitalario) para el estudio nacional sobre la evaluación de las áreas de garantía de calidad en la atención en salud mental pública en Grecia, como parte final de la aplicación de la regulación EEC 815/84 para la reforma psiquiátrica (Madianos 1996, Comisión de la Unión Europea 1995). Es innecesario decir que el entorno físico del hospital psiquiátrico público antes del inicio de este programa de reforma psiquiátrica, que era inaceptable y anacrónico, fue objeto de amplias críticas (Comisión de la Unión Europea 1995).

La construcción de un instrumento nuevo fue resultado del escepticismo sobre la adecuación cultural de instrumentos similares diseñados para su uso en otros países (Janka y Chandrashecar 1993, Srebnik et al. 1997). Esta es una cuestión bastante básica para ser tratada por investigadores en este campo. Se consideró, de este modo, que el desarrollo de un instrumento original era esencial para este estudio. Así, el programa CONDIT fue el resultado de una interacción entre investigadores y clínicos en campos socioculturales comunes.

Se probó que eran satisfactorias las propiedades psicométricas en términos de fiabilidad inter-examinadores y consistencia interna, así como de validez concurrente, de modo que el programa CONDIT es un instrumento bastante fiable y válido. Los ítems de la escala en sus dos Secciones, de Departamento y de planta, cubren todos los componentes básicos del entorno físico de un centro asistencial para pacientes psiquiátricos hospitalizados. El programa parece ser completo y fácil de utilizar por un examinador entrenado en la disciplina. De acuerdo con Brugha y Lindsay (Brugha y Lindsay 1996), la bibliografía existente sobre instrumentación en el área de evaluación de calidad de la estructura es todavía limitada, con pocas excepciones (Janka y Chandrashecar 1993). Este estudio, basado en evaluaciones nacionales sobre la calidad del cuidado psiquiátrico del paciente hospitalizado, podría servir como otro ejemplo de investigación en este campo.

En referencia a la calidad del perfil de la estructura física de los hospitales psiquiátricos públicos, dos hospitales (uno en Atenas, el más grande de Grecia, con 1.700 camas, y otro en la isla de Corfú, el hospital más viejo de los Balcanes, creado en 1835), todavía muestran una calidad pobre del entorno físico, a pesar de los distintos esfuerzos para su 
renovación (Madianos 1996, Comisión de la Unión Europea 1995). En el otro extremo se encontró que el hospital psiquiátrico de Tesalónica, en la ciudad de Tesalónica, reunía estándares muy altos de calidad de estructura física. Esto es debido a las buenas prácticas de dirección y al espíritu de equipo en varios Departamentos (Madianos 1996). Sorprendentemente, las puntuaciones medias totales CONDIT en los Departamentos del hospital psiquiátrico de Leros fueron similares a las puntuaciones medias globales de los Departamentos de los nueve hospitales psiquiátricos. En las puntuaciones de las plantas, se encontró que la puntuación media global CONDIT de las 17 plantas del hospital psiquiátrico de Leros eran las más altas entre las otras 121 Plantas de todos los hospitales psiquiátricos de Grecia. Este es un final más bien feliz de un triste caso que se hizo público. Refleja los tremendos esfuerzos del personal local, limitado en número, y del trabajo infatigable de los equipos de voluntarios, griegos y extranjeros, para la reconstrucción de este manicomio anacrónico, transformándolo en un hospital moderno (Madianos y Economou 1990, Bouras et al. 1992, Tsiantis 1995, Madianos 1996, Comisión de la Unión Europea 1995).

En conclusión, parece que es posible el desarrollo de cuestionarios adaptados culturalmente para reunir los requisitos de los estudios sobre calidad del entorno físico de la atención al paciente psiquiátrico hospitalizado mediante la selección cuidadosa de los pasos en la instrumentación.

Parece estar cambiando en Grecia la situación global de la atención pública psiquiátrica del paciente hospitalizado, en el área crucial del entorno material, lo que tiene implicaciones clínicas y terapéuticas tanto para el paciente como para la plantilla, como se ha visto con el ejemplo ilustrativo del Hospital Psiquiátrico de Leros.
Las investigaciones futuras sobre calidad en esta área monitorizarán el progreso de este cambio.

\section{Agradecimientos}

Los datos expuestos en este artículo son parte de una investigación llevada a cabo por la Unidad de Monitorización y Evaluación de los Servicios de Salud Mental, financiada con becas de la Comisión de la Unión Europea ("Directorate General V").

\section{Bibliografía}

BOURAS, N., WEBB, Y., CLIFFORD, P., PAPADATOS, J., ZOUNI, M. A needs survey among patients in Leros asylum. Brit J Psychiatry 161, 75-79, 1992.

BRUGHA, T.S., LINDSAY, F. Quality of mental health service care: The forgotten pathway from process to outcome. Soc Psychiat Psychiat Epi 31, 89-98, 1996.

CAHN, C., RICHMAN, A. Quality assurance in psychiatry. Can J Psychiatry 30, 148-152, 1985.

COHEN, J. A coefficient of agreement for nominal scales. Educational Psychological Measurement 20, 36-37, 1960.

COHEN, L.H. Research on mental health quality assurance. In: Striker, G., Rodriguez, A.R. eds. Handbook of Quality Assurance in Mental Health. New York: Plenum, 65-79, 1988.

COMMISION OF THE EUROPEAN COMMUNITIES. Report on public mental health reform in Greece by a group of experts. Brussels: CEC, 1984.

COMMISION OF EUROPEAN UNION. Final Report on the implementation of Council Regulation (EEC) 815/84 on Exceptional Financial Support in Favour of Greece in the Social field Brussels: E.C. Directorate General V, 1995.

CONNEL, M.L. Monitoring quality assurance in psychiatry. Amer J Psychiat 147, 1100-1101, 1990.

DAVIS, D., FONG, M. Measuring outcomes in psychiatry: an inpatient model. J Quality Improvement 22, 125-133, 1996. 
DONABEDIAN, A. Criteria and standards for quality assessment and monitoring. Quality Review Bull 12, 99$108,1986$.

DONABEDIAN, A. Evaluating the quality of medical care. Milbank Mem, Fund Quart 44, 166-206, 1966.

DONABEDIAN, A. The criteria and standards of quality: explorations in quality and monitoring series vol II. Ann Arbor: Michigan Health Administration Press, 1982.

DONABEDIAN, A. The definition of quality and approaches to its assessment. Ann Arbor Michigan Health Admin, Press, 1980

DONABEDIAN, A. The quality of care, how can it be assessed? JAMA 260, 1743-1748, 1988,

DONABEDIAN, A. The role of outcomes in quality assessment and assurance. Quality Review Bull 18, 356360, 1992.

EPPEL, A., FUYARCHAK, C., PHELPS, D., TERSIGNI PHELAN, A. A comprehensive and practical quality assurance programme for community mental health services. Can J Psychiatry 36, 102-106, 1991.

FAUMAN, M. Quality assurance and psychiatric practice: A review. Amer J Psychiatry 146, 1121-1130, 1989.

FLEISS, J. Statistical methods for rates and proportions: New York: J Wiley, 1973.

JANKA, A., CHANDRASHECAR, C.R. Catalogue of assessment instruments used in the studies co-ordinated by the WHO mental health programme. Geneva: WHO/MNH/ $92,5,1993$.

JENKINS, R. Towards a system of outcome indicators for mental health care. Brit J Psychiatry 177, 500-514, 1990.

LANGLEY, D.G. Quality assurance in psychiatric treatment. Psychiatric Hospital 11, 13-17, 1980.

LAVENDER, A., LEIPER, R., PILLINGS, S., CLIFFORD, P. Quality assurance in mental health: The QUARTZ system. Brit J Clin Psychiatry 33, 451-467, 1994.

LIPTZIN, B. Quality assurance and psychiatric practice A review. Amer J Psychiatry 131, 1374-1377, 1974.

MADIANOS, M., ECONOMOU, M. Institutional care and rehabilitation in Greece: A general view and the case of Leros. In: Stefanis C., Rabavilas A., Soldatos C. eds. Psychiatry: A world perspective Vol 4. Amsterdam: Elsevier, 629-634, 1990.

MADIANOS, M., YFANTOPOULOS, J., KAPRINIS, G., BALlAS, C., CHRISTODOULOU, G. Decentralization of mental health services in Greece (1979/821989/92). In: Christodoulou, G., Kontaxakis, V. eds. Topics in Preventive Psychiatry. Basel: Karger, 103-115, 1994.
MADIANOS, M., YFANTOPOULOS, J. The First Monitoring Report on the Greek Psychiatric Reform: EEC Regulation 815/84. Brussels: CEC V Directorate General, 1990.

MADIANOS, M. Final Monitoring Report on the progress of the implementation of the psychiatric Reform Programme in Greece under Regulation N. ${ }^{\circ} 815 / 84$. Athens: Monitoring Evaluation of Mental Health Services Unit, UMHR, 1996.

MADIANOS, M. Mental Illness and mental health in Greece. Public Health Rev 11, 73-93, 1983.

MATTSON, M.R. Quality assurance: A literature review of a changing field. Hosp Community Psychiatry 35, 605-615, 1984.

NORUSIS, M. SPSS for Windows. Release 6.0. Chicago: SPSS, 1983.

SCHULBERG, H.C. Quality of care standards and professional norms. Amer J Psychiatry 133, 1017-1051, 1976.

SHERMAN, P. Simple quality assurance measures. Evaluation and Program Planning 10, 227-229, 1987.

SREBNIK, D., HENDRYX, M., STEVENSON, M., CARERLY, S., DYCK, D., CAUCE, A.M. Development of outcome indicators for monitoring the quality of public mental health care. Psychiatric Services 18, 903-909, 1997.

STEFANIS, C., MADIANOS, M., GITTELMAN, M. Recent advances in the care, treatment and rehabilitation of chronically mentally ill in Greece. Hospital Community Psychiatry 37, 1041-1044, 1986.

TICHLER, G.L., ASTRACHAN, B.M. Quality assurance in mental health: peer and utilization review. Rockville Md: US Dept of Health and Human Services, 1982.

TSIANTIS, J. The children of Leros PIKPA. Brit J. Psychiatry 167 suppl 28, 1995.

TUGVEL, A. Methodological perspective on process measures of the quality of medical care. Clin Invest Med 2, 113-121, 1979.

WELLS, K.B., BROOK, R.H. The quality of mental health services: past present and future. London: Plenum, 1988.

ZUSMAN, J. Quality assurance in mental health care. Hosp Community Psychiatry 39, 1266-1290, 1988.

Dirección para correspondencia :

Professor Michael G. Madianos

Zografou Community Mental Health Centre - Social Psychiatry Unit University of Athens 44 Davaki - Pindou 15773 Zografou

Athens

GREECE 